\title{
AFFINE SYMMETRIES OF THE EQUIVARIANT QUANTUM COHOMOLOGY RING OF RATIONAL HOMOGENEOUS SPACES
}

\author{
Pierre-Emmanuel Chaput, Laurent Manivel, and Nicolas Perrin
}

\begin{abstract}
Let $X$ be a rational homogeneous space and let $Q H^{*}(X)_{l o c}^{\times}$be the group of invertible elements in the small quantum cohomology ring of $X$ localised in the quantum parameters. We generalise results of [2] and realise explicitly the map $\pi_{1}(\operatorname{Aut}(X)) \rightarrow$ $Q H^{*}(X)_{l o c}^{\times}$described in [14]. We even prove that this map is an embedding and realise it in the equivariant quantum cohomology ring $Q H_{T}^{*}(X)_{l o c}^{\times}$. We give explicit formulas for the product by these elements.

The proof relies on a generalisation, to a quotient of the equivariant homology ring of the affine Grassmannian, of a formula proved by Peter Magyar [8]. It also uses Peterson's unpublished result [11] — recently proved by Lam and Shimozono in [7] on the comparison between the equivariant homology ring of the affine Grassmannian and the equivariant quantum cohomology ring.
\end{abstract}

\section{Introduction}

For $G$ a semisimple simply connected algebraic group, the center $Z$ of $G$ has several interpretations. It may be canonically identified to the fundamental group $\pi_{1}\left(G^{\text {ad }}\right)$ of the adjoint group $G^{\text {ad }}=G / Z$. Another description is

$$
Z \simeq P^{\vee} / Q^{\vee}
$$

where $P^{\vee}$ and $Q^{\vee}$ are the coweight and coroot lattices (see for example [1]). Nice representatives of this quotient in $P^{\vee}$ are given by the opposites of the minuscule fundamental coweights $\left(-\varpi_{i}^{\vee}\right)_{i \in I_{m}}$ (recall that a dominant coweight $\lambda$ is minuscule if $\langle\lambda, \alpha\rangle=0$ or 1 for any positive root $\alpha$ ). Here $I_{m}$ denotes the subset of the set $I$ of vertices of the Dynkin diagram of $G$ parametrising the minuscule coweights.

The group $Z$ can also be seen as the stabiliser, in the affine Weyl group $W_{\text {aff }}$, of the fundamental alcove (see [7, Page 16] or [8, Page 5]). By composition with the natural map $W_{\text {aff }} \rightarrow W$ to the finite Weyl group of $G$, one realises $Z$ as a subgroup of $W$. This subgroup is given by the elements $\left(v_{i}\right)_{i \in I_{m}}$, where $v_{i}$ is the smallest element of $W$ such that $v_{i} \varpi_{i}^{\vee}=w_{0} \varpi_{i}^{\vee}$, where $w_{0}$ denotes the longest element in $W$ (see $[7$, Page 16] for example).

In a different context, Seidel proved in [14] that the fundamental group of the group of Hamiltonian symplectomorphisms of a symplectic variety $X$ can be mapped to the group $Q H^{*}(X)_{l o c}^{\times}$of invertible elements of the quantum cohomology ring localised in the quantum parameters (Seidel's construction has coefficients in $\mathbb{Z} / 2 \mathbb{Z}$; here we use

Received by the editors December 19, 2007.

Key words: equivariant quantum cohomology, homogeneous space, Schubert calculus, Gromov-Witten invariant.

Mathematics Subject Classification: 14M15, 14N35 
the definition in [9] which has integer coefficients). When $X=G / P$ is a rational homogeneous space, the natural projective structure of $X$ induces a symplectic structure preserved by $G^{a d}$ (recall that the center $Z$ of $G$ acts trivially on $X$ ), and we get a map

$$
Z=\pi_{1}\left(G^{a d}\right) \longrightarrow Q H^{*}(G / P)_{l o c}^{\times}
$$

which we call Seidel's representation. According to [9], it is hard to describe this map explicitly. This has been done by A. Postnikov for Grassmannians and the variety of complete flags in type $A$ (see [12] and [13]). In [2], we described explicitly Seidel's representation when $X=G / P$ is a minuscule or cominuscule homogeneous space; in particular we proved it is faithful. In this note we extend this result to all homogeneous spaces. Moreover we provide an explicit formula for the product by a class in the image of Seidel's representation, in the more general setting of the equivariant quantum cohomology ring $Q H_{T}^{*}(X)_{l o c}$.

To the parabolic subgroup $P$ of $G$, containing a fixed Borel subgroup, we associate the set $I_{P}$ of vertices of the Dynkin diagram defining $P$ (with the convention that if $P$ is the Borel subgroup itself, $I_{P}=I$ is the whole set of vertices of the Dynkin diagram). For $w \in W$, we denote by $\sigma^{P}(w)$ the Schubert class induced by $w$ in $H^{2 l(w)}(G / P, \mathbb{Z})$ and by $\eta_{P}$ the natural surjection $Q^{\vee} \rightarrow Q^{\vee} / Q_{P}^{\vee}$ from the coroot lattice to its quotient by the coroot lattice of $P$. We prove:

Theorem 1. For any $i \in I_{m}$ and any $w \in W$, we have in $Q H_{T}^{*}(G / P)_{l o c}$ :

$$
\sigma^{P}\left(v_{i}\right) * \sigma^{P}(w)=q_{\eta_{P}\left(\varpi_{i}^{\vee}-w^{-1}\left(\varpi_{i}^{\vee}\right)\right)} \sigma^{P}\left(v_{i} w\right) .
$$

Theorem 2. Seidel's representation of the group $\pi_{1}\left(G^{\text {ad }}\right)$ in $Q H^{*}(G / P)_{l o c}^{\times}$is given, for $i \in I_{m}$, by $-\omega_{i} \mapsto \sigma^{P}\left(v_{i}\right)$.

For the complete flag variety $G / B$, Theorem 1 follows rather directly from a factorization theorem in the affine Grassmannian due to Magyar, and Peterson's comparison theorem between the equivariant homology of the affine Grassmannian, and the quantum cohomology of $G / B$ (see Remark 3.18). In order to prove Theorem 1 for arbitrary flag varieties $G / P$, we will need to extend Magyar's result in a suitable way. This extension will rely on the introduction of certain variants of the tools introduced in [11] and [7], and an important part of our work will consist in checking that some of the key statements in these papers still hold in our extended setting. Once our generalized Magyar's formula, Proposition 3.16, is established, the proof of Theorem 1 in 3.5 readily follows.

\section{Peterson's map and Magyar's factorisation formula}

2.1. Affine Grassmannian, affine Weyl group and extended affine Weyl group. Let us denote by $\Omega K$ the affine Grassmannian associated to the group $G$. This is an ind-variety which can be defined as follows. Let $K$ be a maximal compact subgroup of $G$; as a set, the affine Grassmannian is the set of functions $f: S^{1} \rightarrow K$, such that $f(1)=1_{K}$ and $f$ can be extended to a meromorphic function $f: D \rightarrow G$ on the closed unit disk with poles only at the origin (see for example [8] for more details). The affine Grassmannian $\Omega K$ may also be seen as the quotient $G(\mathbb{C}((t))) / G(\mathbb{C}[[t]])$.

The affine Weyl group $W_{\text {aff }}$ is defined as the semidirect product of the Weyl group $W$ by the coroot lattice $Q^{\vee}$ (see [8] of [7] for more details). In the same way one 
defines the extended affine Weyl group $\widetilde{W}_{\text {aff }}$ as the semidirect product of $W$ by $P^{\vee}$ the coweight lattice (see [8] or [7]). Elements of these groups will be denoted by $w t_{\lambda}$ with $w \in W$ and $\lambda \in P^{\vee}$ or $\lambda \in Q^{\vee}$. We will denote by $P_{-}^{\vee}$ the set of antidominant coweights and by $\widetilde{Q}$ its intersection with the coroot lattice.

Note that $W_{\text {aff }}$ is naturally a subgroup of $\widetilde{W}_{\text {aff }}$, which is normal, and that the quotient $\widetilde{W}_{\text {aff }} / W_{\text {aff }}$ is isomorphic to $P^{\vee} / Q^{\vee}$ and thus to $Z$. The stabiliser of the fundamental alcove defines a natural section $Z \rightarrow \widetilde{W}_{\text {aff }}$ of this quotient (see [8, Page $5]$ ). The opposite of the minuscule coweights $\left(-\varpi_{i}^{\vee}\right)_{i \in I_{m}}$ are representatives of the quotient $P^{\vee} / Q^{\vee}$ in $P^{\vee}$. The image of $-\varpi_{i}^{\vee}$ with $i \in I_{m}$ by this section is the element (see [7, Page 16]):

$$
\tau_{i}:=v_{i} t_{-\varpi_{i}^{\vee}} .
$$

Recall the definition of the affine root lattice $Q_{\mathrm{aff}}=\bigoplus_{i=0}^{n} \mathbb{Z} \alpha_{i}$ where $\alpha_{i}$ are the simple roots associated to the extended (or affine) Dynkin diagram. Recall also the definition of the imaginary root $\delta=\alpha_{0}+\theta$ where $\theta$ is the longest root of the finite root system. For more details on the affine root lattice, see [7]. Recall also the action of the extended Weyl group $\widetilde{W}_{\text {aff }}$ on the affine root lattice given by $w t_{\lambda}(\alpha+n \delta)=$ $w(\alpha)+(n-\langle\lambda, \alpha\rangle) \delta$. It follows that any $\tau \in Z$ induces an automorphism $i \mapsto \tau(i)$ of the Dynkin diagram defined by the formula $\tau\left(\alpha_{i}\right)=\alpha_{\tau(i)}$ for any simple root $\alpha_{i}$.

We already defined the element $v_{i}$; it is also given by $v_{i}=w_{0} w_{0}^{P_{i}}$, where $w_{0}$ denotes the longest element of the Weyl group $W$, and $w_{0}^{P_{i}}$ the longest element of the Weyl group $W_{P_{i}}$ of the maximal parabolic subgroup $P_{i}$ of $G$ associated to the cominuscule simple root $\alpha_{i}{ }^{2}$. The element $v_{i}$ is also the longest element in $W^{P_{i}}$, the set of minimal length representatives of the quotient $W / W_{P_{i}}$. This element is well understood, in particular, we shall use the following fact. Recall that the Weyl involution $f$ is defined on roots by $\alpha \mapsto-w_{0}(\alpha)$ and induces an involution on the set of simple roots, that we also denote by $f$.

Fact 2.1. (८) We have $v_{i}^{-1}=v_{f(i)}$.

(2i) Let $\alpha$ be a positive root, then $v_{i}(\alpha)$ is positive if and only if $\left\langle\varpi_{i}^{\vee}, \alpha\right\rangle=0$.

2.2. Equivariant homology. We will be interested in the equivariant homology ring $H_{*}^{T}(\Omega K)$ of $\Omega K$ for a maximal torus $T$ in $G$. We refer to [8] for details. Let $S=H_{T}^{*}(\mathrm{pt})$, then $H_{*}^{T}(\Omega K)$ is a free $S$-module with basis $\left(\xi_{x}\right)_{x \in W_{\text {aff }}^{-}}$where $\xi_{x}$ is the class of a Schubert variety in $\Omega K$ and $W_{\text {aff }}^{-}$is the set of minimal length representatives of $W_{\text {aff }} / W$ in $W_{\text {aff }}$. Note that we will assume, following [6, Page 4], that $T$ does not contain the rotation action of $S^{1}$ on $\Omega K$ (considered as a set of loops). This implies that the image in $S$ of the imaginary root $\delta=\theta+\alpha_{0}$ is zero.

Let us denote by $R$ the root system, by $R^{+}$the set of positive roots and by $R_{P}^{+}$ the set of positive roots in $P$. Define the subset $\left(W^{P}\right)_{\text {aff }}$ of $W_{\text {aff }}$ by

$$
\left(W^{P}\right)_{\text {aff }}=\left\{w t_{\lambda} \in W_{\text {aff }} / \forall \alpha \in R_{P}^{+},\langle\lambda, \alpha\rangle=\left\{\begin{array}{ll}
0 & \text { if } w(\alpha)>0 \\
-1 & \text { if } w(\alpha)<0
\end{array}\right\} .\right.
$$

Recall that we have

$$
W_{\mathrm{aff}}=-\left\{w t_{\lambda} \in W_{\mathrm{aff}} / \forall i,\left\langle\lambda, \alpha_{i}\right\rangle=0 \Longrightarrow w\left(\alpha_{i}\right)>0\right\} .
$$

\footnotetext{
${ }^{2}$ Recall that these elements $w_{0}^{P_{i}}$ define by $\sigma^{P_{i}}(u) \mapsto \sigma^{P_{i}}\left(w_{0}^{P_{i}} u\right)$ the strange duality in $Q H_{l o c}^{*}\left(G / P_{i}\right)$, cf. [2] and [3]
} 
Lam and Shimozono proved that

$$
J_{P}=\bigoplus_{x \in W_{\text {aff }} \backslash\left(W^{P}\right)_{\text {aff }}} S \xi_{x}
$$

is an ideal in $H_{*}^{T}(\Omega K)$. Moreover, they defined a map $\pi_{P}: W_{\text {aff }} \rightarrow\left(W^{P}\right)_{\text {aff }}$ by proving that for any $x \in W_{\text {aff }}$, there exists a unique factorisation $x=x_{1} x_{2}$ with $x_{1} \in\left(W^{P}\right)_{\text {aff }}$ and $x_{2} \in\left(W_{P}\right)_{\text {aff }}$ (the affine group associated to $\left.W_{P}\right)$. The map $\pi_{P}$ is defined by $x \mapsto x_{1}$. We have the following proposition (see [7, Proposition 10.11]):

Proposition 2.2. Let $x \in W_{\mathrm{aff}}^{-} \cap\left(W^{P}\right)_{\text {aff }}$ and $\lambda \in \widetilde{Q}$. Then $x \pi_{P}\left(t_{\lambda}\right) \in W_{\mathrm{aff}}^{-} \cap\left(W^{P}\right)_{\mathrm{aff}}$ and we have:

$$
\xi_{x} \xi_{\pi_{P}\left(t_{\lambda}\right)}=\xi_{x \pi_{P}\left(t_{\lambda}\right)} \bmod J_{P}
$$

In particular, we may quotient and localise the equivariant homology ring as follows, and define

$$
H_{*}^{T}(\Omega K)_{P}:=\left(H_{*}^{T}(\Omega K) / J_{P}\right)\left[\xi_{\pi_{P}\left(t_{\lambda}\right)}^{-1}, \lambda \in \widetilde{Q}\right] .
$$

2.3. Equivariant quantum cohomology. On the other hand, we consider the equivariant quantum cohomology ring $Q H_{T}^{*}(G / P)$. For details we refer to [10] and [7]. This ring is a free $S\left[q_{i}, i \in I_{P}\right]$-module with basis given by the Schubert classes $\left(\sigma^{P}(w)\right)_{w \in W^{P}}$ (where $W^{P}$ is the set of minimal coset representatives of $W / W_{P}$ and $W_{P}$ is the Weyl group of $P$ ). We localise this ring as follows:

$$
Q H_{T}^{*}(G / P)_{l o c}:=Q H_{T}^{*}(G / P)\left[q_{i}^{-1}, i \in I_{P}\right] .
$$

Lam and Shimozono [7, Theorem 10.16] proved the so called Peterson theorem (see also [11]):

Theorem 3. The map $\psi_{P}: H_{*}^{T}(\Omega K)_{P} \rightarrow Q H_{T}^{*}(G / P)_{l o c}$ defined by

$$
\xi_{w \pi_{P}\left(t_{\lambda}\right)} \xi_{\pi_{P}\left(t_{\mu}\right)}^{-1} \mapsto q_{\eta_{P}(\lambda-\mu)} \sigma^{P}(w),
$$

for $w \in W^{P}$ and $\lambda, \mu \in \widetilde{Q}$, is an isomorphism of $S$-algebras.

2.4. Magyar's factorisation formula. Denote by $\widetilde{W}_{\text {aff }}^{-}$the set of minimal length representatives of $\widetilde{W}_{\text {aff }} / W$ in $\widetilde{W}_{\text {aff }}$. An element $x \in \widetilde{W}_{\text {aff }}^{-}$can be uniquely written as $x=\tau_{i} \widehat{x}$ where $\widehat{x} \in W_{\text {aff }}^{-}$and $i \in I_{m}$. Magyar proved the following formula (see $[8$, Theorem A], the length condition $l(x)+l\left(t_{\lambda}\right)=l\left(x t_{\lambda}\right)$ being always satisfied for $x$ in $W_{\text {aff }}^{-}$and $\left.\lambda \in P_{-}^{\vee}\right)$ :

Theorem 4. Let $\lambda$ be antidominant in $P^{\vee}$ and let $x$ and $y$ in $\widetilde{W}_{\mathrm{aff}}^{-}$. Assume that $x t_{\lambda}=y$. Then

$$
\xi_{\widehat{x}} \xi_{\widehat{\lambda_{\lambda}}}=\xi_{\widehat{y}} \quad \text { in } H_{*}^{T}(\Omega K)
$$

In order to prove Theorem 1 we will need a more general formula that will be proven in the next section. However Magyar's formula was a guide for our proof, and it is sufficient to establish Theorem 1 for $G / B$ (see Remark 3.18). 


\section{Symmetries in the quantum cohomology}

Instead of Magyar's formula, which is true in the full equivariant homology ring $H_{*}^{T}(\Omega K)$, we prove a more general formula in the quotient ring $H_{*}^{T}(\Omega K) / J_{P}$. Theorem 1 will be a straightforward application.

We start with the definition of an extended nil Hecke ring $\widetilde{\mathbb{A}}_{\text {Aff }}$, acting on the equivariant homology ring $H_{*}^{T}\left(\Omega K^{\text {ad }}\right)$ of the extended affine Grassmannian $\Omega K^{\text {ad }}$ (see section 3.2). This action extends the known action of $\mathbb{A}_{\mathrm{Aff}}$ on $H_{*}^{T}(\Omega K)$.

3.1. Extended nil Hecke ring. Because of the natural inclusion of $Z$ in $\widetilde{W}_{\text {aff }}$, we can let $Z$ act on the weight lattice; the result of the action of $\tau \in Z$ on a weight $\lambda$ will be simply denoted $\tau(\lambda)$. We have already observed that $\tau\left(\alpha_{i}\right)=\alpha_{\tau(i)}$. Recall from [7, Section 6.1] the definition of the affine nil Hecke ring. It is a non-commutative ring with generators $A_{i}$ for $i \in I_{\mathrm{Aff}}$ and $\lambda$ in the weight lattice, subject to the following relations:

$$
\begin{array}{rll}
\lambda \mu & =\mu \lambda \\
A_{i} \lambda & =\left(s_{i} \cdot \lambda\right) A_{i}+\left\langle\lambda, \alpha_{i}^{\vee}\right\rangle \cdot 1 & \\
A_{i} A_{i} & =0 & \\
A_{i} A_{j} A_{i} \cdots & =A_{j} A_{i} A_{j} \cdots \quad \text { if } s_{i} s_{j} s_{i} \cdots=s_{j} s_{i} s_{j} \cdots
\end{array}
$$

(in the last line, there are the same numbers of factors in all the products). Note that the above set of relations is invariant under the action of $\tau \in Z$, so that $\tau$ yields an algebra automorphism of $\mathbb{A}_{\text {Aff }}$.

Definition 3.1. The extended nil Hecke ring $\widetilde{\mathbb{A}}_{\text {Aff }}$ is the smashed product $Z \ltimes \mathbb{A}_{\text {Aff }}$ of $\mathbb{A}_{\text {Aff }}$ by $Z$. As a $\mathbb{Z}$-module, it is just the tensor product $Z \otimes \mathbb{A}_{\mathrm{Aff}}$. The multiplication is defined by

$$
(\tau \otimes a)(\sigma \otimes b)=\tau \sigma \otimes \sigma^{-1}(a) b .
$$

In other words, $\widetilde{\mathbb{A}}_{\mathrm{Aff}}$ is generated by $Z$ and $\mathbb{A}_{\mathrm{Aff}}$, with the relations $\tau a=\tau(a) \tau$.

Proposition 3.2. Let $x \in \widetilde{W}_{\mathrm{aff}}$ and write $x=\tau \widehat{x}$ with $\tau \in Z$ and $\widehat{x} \in W_{\mathrm{aff}}$. Then the map $x \mapsto \tau \otimes \widehat{x}$ extends the inclusion of $W_{\mathrm{aff}}$ in $\mathbb{A}_{\mathrm{Aff}}$ to a multiplicative inclusion

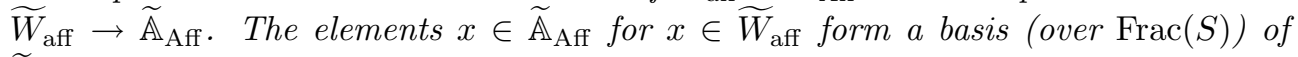
$\widetilde{\mathbb{A}}_{\text {Aff }}$.

Proof. We only need to prove that this map is multiplicative. For $x=\tau \widehat{x}$ and $y=\sigma \widehat{y}$, we have $x y=\tau \sigma \sigma^{-1}(\widehat{x}) \widehat{y}$ in $\widetilde{W}_{\text {aff }}$ and the result follows. Since the elements of $\mathbb{A}_{\mathrm{Aff}}$ defined by $\widehat{x} \in W_{\text {aff }}$ form a basis of $\mathbb{A}_{\text {Aff }}$ over $\operatorname{Frac}(S)$, we can deduce the result for $\widetilde{\mathbb{A}}_{\text {Aff }}$.

3.2. Equivariant homology of $\Omega K^{\text {ad }}$. Recall from [8, Page 17] the definition of the variety $\Omega K^{\text {ad }}$ of based loops with values in the adjoint group $K^{\text {ad }}=K / Z$. The variety $\Omega K^{\text {ad }}$ has its connected components indexed by $Z$. There is an action of $T$ on $\Omega K^{\text {ad }}$ and the fixed points for this action are indexed by $\widetilde{W}_{\text {aff }} / W$. Define the space $L K^{\text {ad }}$ of all loops in $K^{\text {ad }}=K / Z$. We may also (as for $\Omega K$, see [8]) see $\Omega K^{\text {ad }}$ as the quotient $L K^{\text {ad }} / K^{\text {ad }}$ by writing any loop $f \in L K^{\text {ad }}$ as $f(t)=f(t) f(1)^{-1} f(1) \in \Omega K^{\text {ad }} \cdot K^{\text {ad }}$. In particular, we have a left action of $L K^{\text {ad }}$ on $\Omega K^{\text {ad }}$. The group $Z$ can be realised as the subgroup of $L K^{\text {ad }}$ consisting of the points defined by the loops $\left(t \mapsto v_{i} \exp \left(-2 i \pi t \varpi_{i}^{\vee}\right)\right)$ 
(see [8, Page 6]). This together with the classical realisation of $W_{\text {aff }}$ in $L K^{\text {ad }}$ gives a realisation of the extended Weyl group $\widetilde{W}_{\text {aff }}$ in $L K^{\text {ad }}$. The corresponding elements are precisely the fixed points of the $T$-action.

The equivariant cohomology ring $H_{*}^{T}\left(\Omega K^{\text {ad }}\right)$ has a basis indexed by $\widetilde{W}_{\text {aff }}^{-}$: if $\xi_{\widehat{x}}$, with $\widehat{x} \in W_{\text {aff }}^{-}$, is a class in $H_{*}^{T}(\Omega K)$ and $\tau$ is an element in $Z$, then the translation by $\tau \in L K^{\text {ad }}$ in $\Omega K^{\text {ad }}$ defines a class $\widetilde{\xi}_{\tau \widehat{x}}=\tau \cdot \xi_{\widehat{x}}$ in $H_{*}^{T}\left(\Omega K^{\text {ad }}\right)$. Note that $\xi_{\tau}$ is simply the class $[\tau]$. Following [8, Paragraph 1.2], we have for any $\tau \in Z$ and any $\widehat{x} \in W_{\text {aff }}^{-}$:

$$
\xi_{\tau} \xi_{\widehat{x}}=\xi_{\widehat{x}} \xi_{\tau} .
$$

The ring $H_{*}^{T}\left(\Omega K^{\mathrm{ad}}\right)$ is thus the tensor product ring $Z \otimes H_{*}^{T}(\Omega K)$ : the product is defined by

$$
\left(\tau \otimes \xi_{\widehat{x}}\right)\left(\sigma \otimes \xi_{\widehat{y}}\right)=\tau \sigma \otimes \xi_{\widehat{x}} \xi_{\widehat{y}} .
$$

We may define operators $A_{i}$ acting on the ring $H_{*}^{T}\left(\Omega K^{\text {ad }}\right)$, in the same way as they are usually defined as operators on $H_{*}^{T}(\Omega K)$. That is, consider $E \rightarrow B$ the universal principal $L K^{\text {ad }}$-bundle. Then $L K^{\text {ad }}$ acts on the right on $E \times \Omega K^{\text {ad }}$ by $f \cdot\left(e, f^{\prime}\right)=\left(e f^{-1}, f f^{\prime}\right)$. In particular, $T$ acts on $E \times \Omega K^{\text {ad }}$ and we may consider the quotient $E \times \times^{T} \Omega K^{\text {ad }}$ of $E \times \Omega K^{\text {ad }}$ by $T$. The equivariant cohomology is defined by $H_{T}^{*}\left(\Omega K^{\text {ad }}\right)=H^{*}\left(E \times{ }^{T} \Omega K^{\text {ad }}\right)$ and the equivariant homology is the dual of that space. Now for any vertex $i \in I \cup\{0\}$ of the extended Dynkin diagram, there exists an associated compact subgroup $K_{i}^{\text {ad }}$ of $L K^{\text {ad }}$ (see for example [8, Page 15] for the subgroup $K_{i}$ of $L K$ the group of loops in $K$; the group $K_{i}^{\text {ad }}$ is the image of $K_{i}$ in $\left.L K^{\text {ad }}\right)$. Consider the map $\pi_{i}: E \times^{T} \Omega K^{\text {ad }} \rightarrow E \times^{K_{i}^{\text {ad }}} \Omega K^{\text {ad }}$ and define the operator $A_{i}$ by integrating on the fibers and pulling-back. By duality this defines an action of $A_{i}$ on $H_{*}^{T}\left(\Omega K^{\text {ad }}\right)$. We may also consider any $s \in S$ as an operator on $H_{*}^{T}\left(\Omega K^{\text {ad }}\right)$. It is enough to do this for a weight $\lambda$, for which the action is defined by intersection with $c_{1}\left(L_{\lambda}\right)$, where $L_{\lambda}$ denotes the line bundle defined by $\lambda$. Finally we define the operator $\tau$ for any $\tau \in Z$ thanks to the translation by $\tau$ in $\Omega K^{\text {ad }}$.

Proposition 3.3. These operators define an action of $\widetilde{\mathbb{A}}_{\mathrm{Aff}}$ on $H_{*}^{T}\left(\Omega K^{\mathrm{ad}}\right)$ extending the action of $\mathbb{A}_{\mathrm{Aff}}$ on $H_{*}^{T}(\Omega K)$. This action can be written as follows:

$$
(\tau \otimes a) \cdot(\sigma \otimes \xi)=\tau \sigma \otimes\left(\sigma^{-1}(a) \cdot \xi\right) .
$$

Proof. If the action is well defined, it certainly extends the action of $\mathbb{A}_{\mathrm{Aff}}$ on $H_{*}^{T}(\Omega K)$. We only need to verify that the commutation relations $\tau A_{i} \tau^{-1}=A_{\tau(i)}$ and $\tau s \tau^{-1}=$ $\tau(s)$ are satisfied.

For the first one, we only need to remark that the conjugate group $\tau\left(K_{i}\right) \tau^{-1}$ is $K_{\tau(i)}$. For the second one, we may suppose that $s$ is a weight $\lambda$. Let $L_{\lambda}^{\prime}$ be the line bundle defined by the twisted action of $T$ on $\mathbb{C}$ given for $t \in T$ by multiplication with $\tau(\lambda)(t)$. The operator $\tau s \tau^{-1}$ is given by the intersection with $c_{1}\left(L_{\lambda}^{\prime}\right)$. This coincides with the intersection with $c_{1}\left(L_{\tau(\lambda)}\right)$.

For $x \in \widetilde{W}_{\text {aff }}$, write $x=\tau \widehat{x}$ with $\tau \in Z$ and $\widehat{x} \in W_{\text {aff }}$. We define the element $\widetilde{A}_{x} \in \widetilde{\mathbb{A}}_{\text {Aff }}$ by $\widetilde{A}_{x}=\tau \otimes A_{\widehat{x}}$. We also define the length of an element $x=\tau \widehat{x}$ as the number of inverted positive real roots, as in [7, Section 10.1], so that $l(x)=l(\widehat{x})$. 
Proposition 3.4. The action of $\widetilde{\mathbb{A}}_{\mathrm{Aff}}$ on $H_{*}^{T}\left(\Omega K^{\mathrm{ad}}\right)$ is given by the following formula

$$
\widetilde{A}_{x} \cdot \widetilde{\xi}_{y}=\left\{\begin{array}{cl}
\widetilde{\xi}_{x y} & \text { if } l(x y)=l(x)+l(y) \text { and } x y \in \widetilde{W}_{\text {aff }}^{-} \\
0 & \text { otherwise. }
\end{array}\right.
$$

Proof. Write $x=\tau \widehat{x}$ and $y=\sigma \widehat{y}$ with $\tau, \sigma \in Z$ and $\widehat{x}, \widehat{y} \in W_{\text {aff. }}$ We have $x y=$ $\tau \sigma \sigma^{-1}(\widehat{x}) \widehat{y}$. We compute

$$
\begin{aligned}
\widetilde{A}_{x} \cdot \widetilde{\xi}_{y} & =\left(\tau \otimes A_{\widehat{x}}\right) \cdot\left(\sigma \otimes \xi_{y}\right) \\
& =\tau \sigma \otimes \sigma^{-1}\left(A_{\widehat{x}}\right) \cdot \xi_{y} \\
& =\tau \sigma \otimes A_{\sigma^{-1}(\widehat{x})} \cdot \xi_{y} .
\end{aligned}
$$

But we know by a result of Kostant and Kumar [4] (see also [7, Section 6.2]) that

$$
A_{\sigma^{-1}(\widehat{x})} \cdot \xi_{\widehat{y}}=\left\{\begin{array}{cl}
\xi_{\sigma^{-1}(\widehat{x}) \widehat{y}} & \text { if } l\left(\sigma^{-1}(\widehat{x}) \widehat{y}\right)=l\left(\sigma^{-1}(\widehat{x})\right)+l(\widehat{y}) \text { and } \sigma^{-1}(\widehat{x}) \widehat{y} \in W_{\text {aff }}^{-} \\
0 & \text { otherwise }
\end{array}\right.
$$

thus we have the equality

$$
\widetilde{A}_{x} \cdot \widetilde{\xi}_{y}=\left\{\begin{array}{cl}
\widetilde{\xi}_{x y} & \text { if } l\left(\sigma^{-1}(\widehat{x}) \widehat{y}\right)=l(\widehat{x})+l(\widehat{y}) \text { and } \sigma^{-1}(\widehat{x}) \widehat{y} \in W_{\text {aff }}^{-} \\
0 & \text { otherwise. }
\end{array}\right.
$$

The equality $l\left(\sigma^{-1}(\widehat{x})\right)=l(\widehat{x})$ comes from the fact that $\sigma$ permutes the simple roots and the formula $l(\widehat{x})=\left|\left\{\alpha \in R_{\text {aff }}^{+} / \widehat{x}(\alpha)<0\right\}\right|$. Moreover, since $x y=\tau \sigma \sigma^{-1}(\widehat{x}) \widehat{y}$, we know that $x y$ is in $\widetilde{W}_{\text {aff }}^{-}$if and only if $\sigma^{-1}(\widehat{x}) \widehat{y}$ is in $W_{\text {aff }}^{-}$. Furthermore, $l\left(\sigma^{-1}(\widehat{x}) \widehat{y}\right)=$ $l(x y)$ and $l(x)+l(y)=l(\widehat{x})+l(\widehat{y})$ so that the condition $l\left(\sigma^{-1}(\widehat{x}) \widehat{y}\right)=l(\widehat{x})+l(\widehat{y})$ is equivalent to the condition $l(x y)=l(x)+l(y)$.

Remark 3.5. There exist, a priori, two actions of the extended affine Weyl group $\widetilde{W}_{\text {aff }}$ on $H_{*}^{T}\left(\Omega K^{\text {ad }}\right)$ : one is given by the embedding of $\widetilde{W}_{\text {aff }}$ in $\widetilde{\mathbb{A}}_{\text {Aff }}$. The other one is simply defined on $H_{*}^{T}\left(\Omega K^{\text {ad }}\right)$ by the operators $R_{x}^{*}$ induced, for $x \in \widetilde{W}_{\text {aff }}$, by the right multiplication $R_{x}$ in $E \times \times^{T} \Omega K^{\text {ad }}$, by $x$ considered as an element of $\subset L K^{\text {ad }}$. This right multiplication operator is well defined because $\widetilde{W}_{\text {aff }}$ normalises $T$. It is a classical (but non trivial) result that these two actions coincide for the classical affine Weyl group $W_{\text {aff }}$ (see [5, Theorem 11.3.9]). Since the action of $\tau$ on a class $\xi_{x}$ is given by $\xi_{\tau x}$ (see Proposition 3.4), which is also the class obtained by right translation on $E^{\text {ad }} \times{ }^{T} \Omega K^{\text {ad }}$, these two actions of $\widetilde{W}_{\text {aff }}$ coincide as well.

3.3. The map $j^{\text {ad }}$. We want to generalise Theorem 6.2 in [7]. For this we need to consider another basis of the ring $H_{*}^{T}\left(\Omega K^{\text {ad }}\right)$ given by $T$-fixed points in $\Omega K^{\text {ad }}$. Recall that these fixed points are indexed by $\widetilde{W}_{\text {aff }} / W$ and that good representatives are given by $P^{\vee}$ by

$$
p_{t_{\lambda}}=(t \mapsto \exp (2 i \pi t \lambda)) \quad \text { for } \lambda \in P^{\vee} .
$$

Furthermore, for $\lambda$ and $\mu$ in $P^{\vee}$, these fixed points satisfy $p_{t_{\lambda}} p_{t_{\mu}}=p_{t_{\lambda} t_{\mu}}$ and the affine Weyl group action gives $x \cdot p_{t_{\lambda}}=p_{x t_{\lambda}}$.

Definition 3.6. Let $\widetilde{\psi}_{t_{\lambda}}$ for $\lambda \in P^{\vee}$ be the element in $H_{*}^{T}\left(\Omega K^{\text {ad }}\right)$ defined by

$$
\widetilde{\psi}_{t_{\lambda}}=i_{\lambda}^{*}: H_{T}^{*}\left(\Omega K^{a d}\right) \rightarrow S,
$$

where $i_{\lambda}$ denotes the inclusion of the $T$-fixed point $p_{\lambda}$ in $\Omega K^{\text {ad }}$. 
The fact that the elements $\widetilde{\psi}_{t_{\lambda}}$, for $\lambda \in P^{\vee}$, form a basis of $H_{*}^{T}\left(\Omega K^{\text {ad }}\right)$ over $\operatorname{Frac}(S)$, comes from the same statement for compact spaces (see [5, Theorem C8, page 537]), and the fact that the extended affine Grassmannian, as well as the affine Grassmannian, is an increasing union of compact $T$-stable finite dimensional Hausdorff subspaces.

Proposition 3.7. We have the following formulas:

$$
\widetilde{\psi}_{t_{\lambda}} \widetilde{\psi}_{t_{\mu}}=\widetilde{\psi}_{t_{\lambda+\mu}} \quad \text { and } \quad x \cdot \widetilde{\psi}_{t_{\lambda}}=\widetilde{\psi}_{x t_{\lambda}}
$$

for any $\lambda$ and $\mu$ in $P^{\vee}$ and any $x$ in $\widetilde{W}_{\text {aff }}$.

Proof. These formulas are immediate consequences of the identities $p_{t_{\lambda}} p_{t_{\mu}}=p_{t_{\lambda} t_{\mu}}$ and $x \cdot p_{t_{\lambda}}=p_{x t_{\lambda}}$.

We are now in position to generalise $[7$, Theorem 6.2$]$. We will denote by $Z_{\widetilde{\mathbb{A}}_{\text {Aff }}}(S)$ the centraliser of $S$ in $\widetilde{\mathbb{A}}_{\text {Aff }}$. We also define the ideal $\widetilde{J}$ in $\widetilde{\mathbb{A}}_{\text {Aff }}$ by

$$
\widetilde{J}=\sum_{w \in W \backslash\{\mathrm{id}\}} \widetilde{\mathbb{A}}_{\mathrm{Aff}}\left(1 \otimes A_{w}\right) .
$$

Proposition 3.8. There is an $S$-algebra isomorphism $j^{\text {ad }}: H_{*}^{T}\left(\Omega K^{\mathrm{ad}}\right) \rightarrow Z_{\widetilde{\mathbb{A}}_{\mathrm{Aff}}}(S)$ such that for any $x$ and $y$ in $\widetilde{W}_{\text {aff }}^{-}$we have $j^{\text {ad }}\left(\widetilde{\xi}_{x}\right)=\widetilde{A}_{x} \bmod \widetilde{J}$ and $j^{\text {ad }}\left(\widetilde{\xi}_{x}\right) \cdot \widetilde{\xi}_{y}=\widetilde{\xi}_{x} \widetilde{\xi}_{y}$.

Proof. This extends the similar result in [6], whose proof we follow. We define $j^{\text {ad }}$ by letting $j^{\text {ad }}\left(\widetilde{\psi}_{t_{\lambda}}\right)=t_{\lambda}$ for $\lambda \in P^{\vee}$, and extending by $\operatorname{Frac}(S)$-linearity. Proposition 3.7 shows that it is an $S$-algebra morphism. The formula $x \cdot \widetilde{\psi}_{t}=\widetilde{\psi}_{x t}$ gives

$$
j^{\operatorname{ad}}\left(\widetilde{\psi}_{t_{\lambda}}\right) \cdot \widetilde{\psi}_{t_{\mu}}=\widetilde{\psi}_{t_{\lambda} t_{\mu}}=\widetilde{\psi}_{t_{\lambda}} \widetilde{\psi}_{t_{\mu}}
$$

which, since the elements $\widetilde{\psi}_{t_{\lambda}}$ for $\lambda \in P^{\vee}$ form a basis of $H_{*}^{T}\left(\Omega K^{\text {ad }}\right)$, implies the identity $j^{\text {ad }}\left(\widetilde{\xi}_{x}\right) \cdot \widetilde{\xi}_{y}=\widetilde{\xi}_{x} \widetilde{\xi}_{y}$.

To prove that the image is contained in $Z_{\widetilde{\mathbb{A}}_{\text {Aff }}}(S)$, consider the action $t_{\lambda} \cdot(\alpha+n \delta)=$ $(\alpha+n \delta-\langle\alpha, \lambda\rangle \delta) t_{\lambda}$ where $\alpha$ is a root and $\delta=\alpha_{0}+\theta$ is the imaginary root. As the image of $\delta$ in $S$ is zero, the commutation relation follows.

We have $j^{\text {ad }}\left(\widetilde{\xi}_{x}\right) \cdot \widetilde{\xi}_{\text {id }}=\widetilde{\xi}_{x} \widetilde{\xi}_{\text {id }}=\widetilde{\xi}_{x}$ for $x \in \widetilde{W}_{\text {aff }}^{-}$. This implies that $j^{\text {ad }}\left(\widetilde{\xi}_{x}\right)=\widetilde{A}_{x}+a$ where $a$ lies in the annihilator of $\widetilde{\xi}_{\text {id }} \in H_{*}^{T}\left(\Omega K^{\text {ad }}\right)$ in $\widetilde{\mathbb{A}}_{\text {Aff }}$. This annihilator is clearly the ideal $\widetilde{J}$.

Finally, let $a=\sum_{x \in \widetilde{W}_{\text {aff }}} a_{x} x$ be an element in $Z_{\widetilde{\mathbb{A}}_{\mathrm{Aff}}}(S)$, then we have for $v \in S$

$$
a v=\sum_{x \in \widetilde{W}_{\mathrm{aff}}} a_{x} x(v) x=v a=\sum_{x \in \widetilde{W}_{\mathrm{aff}}} a_{x} v x .
$$

In particular $a_{x}=0$ for all $x$ such that $x(v) \neq v$ for some $v \in S$. But recall that the action of $w \in W$ is given by $w(\alpha+n \delta)=w(\alpha)+n \delta$ and because $\delta$ is send to zero, this equals $\alpha$ if and only if $w=\mathrm{id}$. In particular $x$ has to be a translation, which exactly means it belongs to the image of $j^{\text {ad }}$. 
3.4. Generalised Magyar's formula. In this subsection, we generalise Magyar's formula to the quotient $H_{*}^{T}(\Omega K) / J_{P}$. This formula, as for Magyar's formula, is a generalisation to coweights of the localisation formula of Proposition 2.2. Let us first introduce some definitions.

Definition 3.9. We define the subset $\left(\widetilde{W}^{P}\right)_{\text {aff }}$ of $\widetilde{W}_{\text {aff }}$ as follows : for $x=\tau \widehat{x}$ with $\tau \in Z$ and $\widehat{x} \in W_{\text {aff }}, x \in\left(\widetilde{W}^{P}\right)_{\text {aff }}$ if $\widehat{x} \in\left(W^{P}\right)_{\text {aff }}$.

Lemma 3.10. Define $\left(R_{P}\right)_{\text {aff }}^{+}=\left\{\alpha+n \delta \in R_{\text {aff }}^{+} / \alpha \in R_{P}\right\}$, then we have

$$
\left(\widetilde{W}^{P}\right)_{\mathrm{aff}}=\left\{x \in \widetilde{W}_{\mathrm{aff}} / x(\beta)>0 \text { for all } \beta \in\left(R_{P}\right)_{\mathrm{aff}}^{+}\right\}
$$

Proof. Recall from [7, Section 10.3], that the condition in the lemma defines $\left(W^{P}\right)_{\text {aff }}$ in $W_{\text {aff }}$. The extension to $\left(\widetilde{W}^{P}\right)_{\text {aff }}$ follows from the fact that $Z$ stabilises the cone of positive roots.

For an element $x \in \widetilde{W}_{\text {aff }}$, we write $x=\tau \widehat{x}$ with $\tau \in Z$ and $\widehat{x} \in W_{\text {aff }}$. We need to compare the properties of $x$ and $\widehat{x}$. We first compare their expressions as products $x=w t_{\lambda}$ with $\lambda \in P^{\vee}$, and $\widehat{x}=v t_{\mu}$ with $\mu \in Q^{\vee}$.

Lemma 3.11. Let $w \in W$ and $\lambda \in P^{\vee}$. Write $\lambda=-\varpi_{i(\lambda)}^{\vee}+\widehat{\lambda}$, where $\hat{\lambda} \in Q^{\vee}$ and $i(\lambda) \in I_{m}$. Then

$$
w t_{\lambda}=\tau_{i(\lambda)} v_{f(i(\lambda))} w t_{\lambda-w^{-1}\left(\varpi_{f(i(\lambda))}^{\vee}\right)},
$$

where $\lambda-w^{-1}\left(\varpi_{f(i(\lambda))}^{\vee}\right)$ is in $Q^{\vee}$.

Proof. We know that there exist an index $j$, an element $u \in W$ and an element $x \in Q^{\vee}$ such that $w t_{\lambda}=\tau_{j} u t_{x}$. We then get the equalities $u=v_{j}^{-1} w$ and $\mu=\lambda-w^{-1}\left(\varpi_{f(j)}\right)$. But $\mu$ has to be in $Q^{\vee}$, hence $f(j)=f(i(\lambda))$ and thus $j=i(\lambda)$.

Let $\lambda \in P^{\vee}$ and $w \in W$. Write $w t_{\lambda}=\tau_{i(\lambda)} v_{f(i(\lambda))} w t_{\lambda-w^{-1}\left(\varpi_{f(i \lambda))}^{\vee}\right)}$ with $\hat{\lambda} \in Q^{\vee}$.

Lemma 3.12. For any positive root $\alpha \in R^{+}$of the finite root system, we have the equality

$$
\chi(w(\alpha)<0)+\langle\lambda, \alpha\rangle=\chi\left(v_{f(i(\lambda))} w(\alpha)<0\right)+\left\langle\lambda-w^{-1}\left(\varpi_{f(i(\lambda))}^{\vee}\right), \alpha\right\rangle,
$$

where $\chi(A)=1$ if $A$ is true and $\chi(A)=0$ otherwise.

Proof. Let us consider the following four cases:

1. $w(\alpha)>0$ and $\left\langle\varpi_{f(i(\lambda))}^{\vee}, w(\alpha)\right\rangle=1$;

2. $w(\alpha)>0$ and $\left\langle\varpi_{f(i(\lambda))}^{\mathrm{V}}, w(\alpha)\right\rangle=0$;

3. $w(\alpha)<0$ and $\left\langle\varpi_{f(i(\lambda))}^{\vee}, w(\alpha)\right\rangle=-1$;

4. $w(\alpha)<0$ and $\left\langle\varpi_{f(i(\lambda))}^{\vee}, w(\alpha)\right\rangle=0$.

According to these cases and setting $u=v_{f(i(\lambda))} w$ and $\mu=\lambda-w^{-1}\left(\varpi_{f(i(\lambda))}^{\vee}\right)$, we have:

1. $u(\alpha)<0$ and $\langle\mu, \alpha\rangle=\langle\lambda, \alpha\rangle-1$;

2. $u(\alpha)>0$ and $\langle\mu, \alpha\rangle=\langle\lambda, \alpha\rangle$;

3. $u(\alpha)>0$ and $\langle\mu, \alpha\rangle=\langle\lambda, \alpha\rangle+1$;

4. $u(\alpha)<0$ and $\langle\mu, \alpha\rangle=\langle\lambda, \alpha\rangle$. 
The statement can then be checked case by case.

This lemma gives a generalisation to the extended affine Weyl group $\widetilde{W}_{\text {aff }}$ of the length formula given in [7, Lemma 3.1]:

Corollary 3.13. Let $w t_{\lambda} \in \widetilde{W}_{\text {aff }}$ with $w \in W$ and $\lambda \in P^{\vee}$. Then

$$
l\left(w t_{\lambda}\right)=\sum_{\alpha \in R^{+}}|\chi(w(\alpha)<0)+\langle\lambda, \alpha\rangle| .
$$

Proof. The formula $w t_{\lambda}=\tau_{i(\lambda)} v_{f(i(\lambda))} w t_{\lambda-w^{-1}\left(\varpi_{f(i(\lambda))}^{\vee}\right)}$ implies that

$$
l\left(w t_{\lambda}\right)=l\left(v_{f(i(\lambda))} w t_{\lambda-w^{-1}\left(\varpi_{f(i(\lambda))}^{\vee}\right)}\right),
$$

and [7, Lemma 3.1] gives the formula

$$
l\left(v_{f(i(\lambda))} w t_{\lambda-w^{-1}\left(\varpi_{f(i(\lambda))}^{\vee}\right)}\right)=\sum_{\alpha \in R^{+}}\left|\chi\left(v_{f(i(\lambda))} w(\alpha)<0\right)+\left\langle\lambda-w^{-1}\left(\varpi_{f(i(\lambda))}^{\vee}\right), \alpha\right\rangle\right| .
$$

We conclude thanks to the previous lemma.

The previous lemma also provides a criterion, for an element $x \in \widetilde{W}_{\text {aff }}$, to be such that $\widehat{x} \in\left(W^{P}\right)_{\text {aff }}$. More precisely let $w t_{\lambda} \in \widetilde{W}_{\text {aff }}$. Write once more $w t_{\lambda}=$ $\tau_{i(\lambda)} v_{f(i(\lambda))} w t_{\lambda-w^{-1}\left(\varpi_{f(i(\lambda))}^{\vee}\right)}$.

Corollary 3.14. The element $v_{f(i(\lambda))} w t_{\lambda-w^{-1}\left(\varpi_{f(i(\lambda))}^{\vee}\right)}$ is in $\left(W^{P}\right)_{\text {aff }}$ if and only if, for any root $\alpha \in R_{P}^{+}$,

$$
\langle\lambda, \alpha\rangle= \begin{cases}0 & \text { if } w(\alpha)>0 \\ -1 & \text { if } w(\alpha)<0\end{cases}
$$

Proof. This condition is exactly the condition for an element $w t_{\lambda} \in W_{\text {aff }}$ to be in $\left(W^{P}\right)_{\text {aff }}$, see Lemma 10.1 in [7]. But remark that this condition is equivalent to

$$
\chi(w(\alpha)<0)+\langle\lambda, \alpha\rangle=0
$$

for all $\alpha \in R_{P}^{+}$. We conclude once more by the previous lemma.

Corollary 3.15. An element $w t_{\lambda}$ with $w \in W$ and $\lambda \in P^{\vee}$ is in $\left(\widetilde{W}^{P}\right)_{\text {aff }}$ if and only if, for any root $\alpha \in R_{P}^{+}$,

$$
\langle\lambda, \alpha\rangle= \begin{cases}0 & \text { if } w(\alpha)>0 \\ -1 & \text { if } w(\alpha)<0\end{cases}
$$

Proof. This is an immediate application of Corollary 3.14.

The map $\pi_{P}: W_{\mathrm{aff}} \rightarrow\left(W^{P}\right)_{\text {aff }}$ extends to $\widetilde{\pi}_{P}: \widetilde{W}_{\mathrm{aff}} \rightarrow\left(\widetilde{W}^{P}\right)_{\text {aff }}$ by setting $\pi_{P}(x)=$ $\tau \pi_{P}(\widehat{x})$ for $x=\tau \widehat{x}$. Many results of [7, Paragraph 10] on $\left(W^{P}\right)$ aff extend readily to $\left(\widetilde{W}^{P}\right)_{\text {aff }}$. This is the case of Lemma 3.1 (see Corollary 3.13), Lemmas 3.2 and 3.3 and Lemmas 10.2 to 10.6. The definition of the extension $\widetilde{\pi}_{P}$ of $\pi_{P}$ ensures that the results of Proposition 10.8 in [7] remain true in our setting. 
Furthermore, we extend the definition of the ideal $J_{P}$ by defining the following ideal $\widetilde{J}_{P}$ of $\widetilde{\mathbb{A}}_{\text {Aff }}$ :

$$
\widetilde{J}_{P}=\sum_{x \in \widetilde{W}_{\text {aff }}^{-} \backslash\left(\widetilde{W}^{P}\right)_{\text {aff }}} S \widetilde{\xi}_{x} .
$$

This is indeed an ideal because $\widetilde{W}_{\text {aff }}^{-}$and $\left(\widetilde{W}^{P}\right)$ aff are stable under left multiplication by $Z$.

Proposition 3.16. For all $x \in \widetilde{W}_{\text {aff }}^{-} \cap\left(\widetilde{W}^{P}\right)_{\text {aff }}$ and $\lambda \in P_{-}^{\vee}$, we have $x \pi_{P}\left(t_{\lambda}\right) \in$ $\widetilde{W}_{\text {aff }}^{-} \cap\left(\widetilde{W}^{P}\right)_{\text {aff }}$ and

$$
\xi_{\widehat{x}} \xi_{\pi_{P}\left(t_{\lambda}\right)}=\xi_{x \widehat{\pi_{P}\left(t_{\lambda}\right)}} \bmod J_{P}
$$

Proof. We follow the proof of Proposition 2.2 by Lam and Shimozono (see [7, Proposition 10.11]). Lemma 10.10 in [7] extends readily to

Lemma 3.17. Let $\lambda \in P^{\vee}$ be antidominant. Then $\left(1 \otimes A_{i}\right) \cdot \widetilde{\xi}_{\pi_{P}\left(t_{\lambda}\right)}=0 \bmod \widetilde{J}_{P}$ for all $i \in I$.

In particular $\widetilde{J} \cdot \xi_{\pi_{P}\left(t_{\lambda}\right)}=0 \bmod \widetilde{J}_{P}$. Now we can apply Proposition 3.8 to get the identity:

$$
\widetilde{\xi}_{x} \widetilde{\xi}_{\pi_{P}\left(t_{\lambda}\right)}=\widetilde{A}_{x} \cdot \widetilde{\xi}_{\pi_{P}\left(t_{\lambda}\right)} \bmod \widetilde{J}_{P}
$$

Let us prove that the product $x \pi_{P}\left(t_{\lambda}\right)$ is length additive. We have seen that Proposition 10.8 of [7] extends to $\widetilde{W}_{\text {aff }}$ and $\left(\widetilde{W}^{P}\right)_{\text {aff }}$. In particular $x=w \pi_{P}\left(t_{\nu}\right)$ for $w \in W^{P}$ and $\nu \in P^{\vee}$ antidominant and we have $x \pi_{P}\left(t_{\lambda}\right)=w \pi_{P}\left(t_{\nu}\right) \pi_{P}\left(t_{\lambda}\right)=$ $w \pi_{P}\left(t_{\nu+\lambda}\right)$. By [7, Lemma 3.3], because $x$ and $x \pi_{P}\left(t_{\lambda}\right)$ are in $\widetilde{W}_{\text {aff }}^{-}$, we have $l(x)=$ $l\left(\pi_{P}\left(t_{\nu}\right)\right)-l(w)$ and $l\left(x \pi_{P}\left(t_{\lambda}\right)\right)=l\left(\pi_{P}\left(t_{\nu+\lambda}\right)\right)-l(w)$. We only need to prove that $l\left(\pi_{P}\left(t_{\nu+\lambda}\right)\right)=l\left(\pi_{P}\left(t_{\nu}\right)\right)+l\left(\pi_{P}(\lambda)\right)$. Because Lemmas 3.3, 10.3 and 10.6 of [7] extend to coweights, the same proof as for coroots in [7] gives the additivity. By proposition 3.4, we thus have the formula $\widetilde{\xi}_{x} \widetilde{\xi}_{\pi_{P}\left(t_{\lambda}\right)}=\widetilde{\xi}_{x \pi_{P}\left(t_{\lambda}\right)} \bmod \widetilde{J}_{P}$. But writing $x=\tau \widehat{x}, \pi_{P}\left(t_{\lambda}\right)=\sigma \widehat{\pi_{P}\left(t_{\lambda}\right)}$ and $x \pi_{P}\left(t_{\lambda}\right)=\tau \sigma \widehat{x \pi_{P}\left(t_{\lambda}\right)}$, this implies the equality $\left(\tau \otimes \xi_{\widehat{x}}\right)\left(\sigma \otimes \xi_{\widehat{\pi_{P}\left(t_{\lambda}\right)}}\right)=\left(\tau \sigma \otimes \xi_{x \pi_{P}\left(t_{\lambda}\right)}\right) \bmod \widetilde{J}_{P}$ and thus $\xi_{\widehat{x}} \xi_{\widehat{\pi_{P}\left(t_{\lambda}\right)}}=\xi_{x \pi_{P}\left(t_{\lambda}\right)} \bmod J_{P}$.

Finally let us prove that $x \pi_{P}\left(t_{\lambda}\right)$ belongs to $\widetilde{W}_{\text {aff }}^{-} \cap\left(\widetilde{W}^{P}\right)_{\text {aff }}$. Since $x \in\left(\widetilde{W}^{P}\right)_{\text {aff }}$, we have $x \pi_{P}\left(t_{\lambda}\right)=\pi_{P}(x) \pi_{P}\left(t_{\lambda}\right)=\pi_{P}\left(x t_{\lambda}\right)$ (from the generalisation of $[7$, Proposition $10.8]$ to $\left.\widetilde{W}_{\text {aff }}\right)$. But $x \in \widetilde{W}_{\text {aff }}^{-}$and $\lambda \in P_{-}^{\vee}$, thus, by the generalisation of [7, Lemma $3.3]$ to $\widetilde{W}_{\text {aff }}$, we get that $x t_{\lambda} \in \widetilde{W}_{\text {aff }}^{-}$. Thanks, once more, to the generalisation of $[7$, Proposition 10.8$]$ to $\widetilde{W}_{\text {aff }}$, we can then deduce the result.

3.5. Application to symmetries. We are now in position to prove Theorem 1 for any $G / P$. Let $w$ be an element of the Weyl group $W$, let $\varpi_{i}^{\vee}$ be a minuscule coweight and let $\mu$ and $\nu$ be in $Q^{\vee}$. We begin with the formulas:

$t_{-\varpi_{i}^{\vee}-\mu}=\tau_{i} v_{f(i)} t_{-\left(\varpi_{i}^{\vee}+\varpi_{f(i)}^{\vee}+\mu\right)}$ and $w t_{-\nu} t_{-\varpi_{i}^{\vee}-\mu}=\tau_{i} v_{f(i)} w t_{-\left(\varpi_{i}^{\vee}+w^{-1}\left(\varpi_{f(i)}^{\vee}\right)+\mu+\nu\right)}$.

Applying the map $\pi_{P}$, we get the equalities

$$
\pi_{P}\left(t_{-\varpi_{i}^{\vee}-\mu}\right)=\tau_{i} \pi_{P}\left(v_{f(i)}\right) \pi_{P}\left(t_{-\left(\varpi_{i}^{\vee}+\varpi_{f(i)}^{\vee}+\mu\right)}\right)
$$


and

$$
\pi_{P}\left(w t_{-\nu} t_{-\varpi_{i}^{\vee}-\mu}\right)=\tau_{i} \pi_{P}\left(v_{f(i)} w\right) \pi_{P}\left(t_{-\left(\varpi_{i}^{\vee}+w^{-1}\left(\varpi_{f(i)}^{\vee}\right)+\mu+\nu\right)}\right) .
$$

For $\mu$ and $\nu$ dominant enough, the element $w t_{-\nu}, v_{f(i)} t_{-\left(\varpi_{i}^{\vee}+\varpi_{f(i)}^{\vee}+\mu\right)}$ and the element $v_{f(i)} w t_{-\left(\varpi_{i}^{\vee}+w^{-1}\left(\varpi_{f(i)}^{\vee}\right)+\mu+\nu\right)}$ belong to $W_{\text {aff }}^{-}$, and their images by $\pi_{P}$ are in $\left(W^{P}\right)_{\text {aff }} \cap$ $W_{\text {aff }}^{-}$. We may thus apply Proposition 3.16, and we obtain that modulo $J_{P}$ the following holds:

$$
\xi_{\pi_{P}(w) \pi_{P}\left(t_{-\nu}\right)} \xi_{\pi_{P}\left(v_{f(i)}\right) \pi_{P}\left(t_{-\left(\varpi_{i}^{\vee}+\varpi_{f(i)}^{\vee}+\mu\right)}\right)} \equiv \xi_{\pi_{P}\left(v_{f(i)} w\right) \pi_{P}\left(t_{-\left(\varpi_{i}^{\vee}+w^{-1}\left(\varpi_{f(i)}^{\vee}+\mu+\nu\right)\right)}\right)} .
$$

Applying Peterson's map of Theorem 3, we get the corresponding formula in the quantum cohomology ring:

$\sigma^{P}(w) q_{-\eta_{P}(\nu)} * \sigma^{P}\left(v_{f(i)}\right) q_{-\eta_{P}\left(\left(\varpi_{i}^{\vee}+\varpi_{f(i)}^{\vee}+\mu\right)\right)}=\sigma^{P}\left(v_{f(i)} w\right) q_{-\eta_{P}\left(\left(\varpi_{i}^{\vee}+w^{-1}\left(\varpi_{f(i)}^{\vee}\right)+\mu+\nu\right)\right)}$,

hence finally:

$$
\sigma^{P}(w) * \sigma^{P}\left(v_{f(i)}\right)=q_{\eta_{P}\left(\varpi_{f(i)}^{\vee}-w^{-1}\left(\varpi_{f(i)}^{\vee}\right)\right)} \sigma^{P}\left(v_{f(i)} w\right) .
$$

This concludes the proof of Theorem 1.

Remark 3.18. As a final remark in this section, let us explain briefly why Magyar's formula is not sufficient to prove Theorem 1 in full generality. The point is that, in order to apply this formula, we need an element $t_{\lambda} \in \widetilde{W}_{\text {aff }}^{-} \cap\left(\widetilde{W}^{P}\right)_{\text {aff }}$. But Corollary 3.14 implies the following fact:

Fact 3.19. A translation $t_{\lambda}$, with $\lambda \in P^{\vee}$, is in $\widetilde{W}_{\text {aff }}^{-} \cap\left(\widetilde{W}^{P}\right)_{\text {aff }}$ if and only if $\lambda \in P_{-}^{\vee}$ and $\langle\lambda, \alpha\rangle=0$ for all $\alpha \in R_{P}^{+}$.

In particular, if $P$ is a maximal parabolic subgroup associated to a cominuscule coweight $\varpi_{i}^{\vee}$ (equivalently, if $I_{P}=\{i\}$ for some $i$ in $I_{m}$ ), this implies that $\lambda$ needs to be a multiple of $\varpi_{i}^{\vee}$. But then Magyar's formula gives the Theorem 1 only for multiples of $v_{f(i)}$. The corresponding symmetries of the quantum cohomology ring are those generated by the quantum product with the punctual class $[\mathrm{pt}]$, which were described in [2]. These symmetries do not generate the full group $Z$ in general.

However, as we already mentionned, if the parabolic subgroup $P$ is a Borel subgroup, there is no restriction on $\lambda \in P_{-}^{\vee}$. In this case our Theorem 1 follows from Magyar's formula.

\section{Seidel's representation}

In this section, we prove Theorem 2. That is, we prove that $\sigma^{P}\left(v_{f(i)}\right)$ is the invertible element $S\left(\omega_{i}^{\vee}\right)$ of the (localized) quantum cohomology ring, corresponding to $-\omega_{i}^{\vee} \in Z=\pi_{1}\left(G^{\text {ad }}\right)$ through Seidel's representation. As we recalled in the introduction, we use the integer-valued definition given in [9, Example 8.6.8]. Let us recall Seidel's construction.

Since $G$ is simply-connected, the coweight lattice parametrise 1-parameter subgroups of $T$, our prefered maximal torus of $G$ (which is contained in $P$ ). Let $\mathbb{C}^{*} \subset T$ correspond to $\omega_{i}^{\vee}$. The image of $S^{1} \subset \mathbb{C}^{*} \subset T \subset G \rightarrow G^{a d}$ defines an element of $\pi_{1}\left(G^{a d}\right)$ which will be denoted $\pi_{1}\left(\omega_{i}^{\vee}\right)$.

We now set $M_{i}=\left(\mathbb{C}^{2}-\{0\}\right) \times \mathbb{C}^{*} G / P$. There is a natural map $\pi: M_{i} \rightarrow$ $\left(\mathbb{C}^{2}-\{0\}\right) / \mathbb{C}^{*} \simeq \mathbb{P}^{1}$. The fibers of $\pi$ are isomorphic with $G / P$. As in [14], we 
denote $T M_{i}^{v}$ the vertical tangent space, that is, the kernel of the differential $d \pi$ in $T M_{i}$. Choose a point $z \in \mathbb{P}^{1}$, and if $S$ is a space of sections of $\pi$, denote by ev $: S \rightarrow \pi^{-1}(z) \simeq G / P$ the evaluation map at $z$. In the following proposition, $k$ denotes the number of roots $\alpha$ in the unipotent radical of $P$ such that $\left\langle\omega_{i}^{\vee}, \alpha\right\rangle>0$.

Proposition 4.1. For any section $s$ of $\pi$, one has $\operatorname{deg}\left(T M_{i}^{v}\right)(s) \geq-k$. Moreover, let $S$ denote the space of sections $s$ of $\pi$ such that $\operatorname{deg}\left(T M_{i}^{v}\right)(s)=-k$. Then ev $_{*}[S] \in$ $H_{*}(G / P)$ identifies via Poincaré duality with $\sigma^{P}\left(v_{i}\right)$.

We postpone the proof to the end of the section. Let us first investigate the geometry of $M_{i}$ in more details. For $\lambda$ a $W_{P}$-invariant element of the root lattice, we denote by $L_{\lambda}$ the associated $G$-linearised bundle on $G / P$. Moreover, if $L$ is any $G$-linearised bundle, we denote $L^{i}$ the bundle $L \times \mathbb{C}^{*}\left(\mathbb{C}^{2}-0\right)$ over $M_{i}$. Let us finally denote $\lambda_{P}=\sum_{\alpha \in \Phi^{+}-\Phi_{P}} \alpha$.

Proposition 4.2. We have $\operatorname{det}\left(T M_{i}^{v}\right)=L_{\lambda_{P}}^{i}$.

Proof. Let $\eta: G / P \times\left(\mathbb{C}^{2}-0\right) \rightarrow M_{i}$ denote the quotient map, and let $p_{1}: G / P \times$ $\left(\mathbb{C}^{2}-0\right) \rightarrow G / P$ denote the first projection. Since $\eta$ is a submersion, it induces an isomorphism of vector bundles $p_{1}^{*} T G / P \simeq \eta^{*} T M_{i}^{v}$. Moreover, this isomorphism commutes with the $\mathbb{C}^{*}$-action, so that it induces an isomorphism $T G / P \times \mathbb{C}^{*}\left(\mathbb{C}^{2}-0\right) \simeq$ $T M_{i}^{v}$. We deduce that $\operatorname{det}\left(T M_{i}^{v}\right) \simeq \operatorname{det}(T G / P) \times \mathbb{C}^{*}\left(\mathbb{C}^{2}-0\right) \simeq L_{\lambda_{P}}^{i}$.

Corollary 4.3. For $s: \mathbb{P}^{1} \rightarrow M_{i}$ any section of $\pi$, one has $\operatorname{deg} s^{*} \operatorname{det}\left(T M_{i}^{v}\right) \geq-k$.

Proof. Let $N=\operatorname{det}\left(T M_{i}^{v}\right) \otimes \pi^{*} O(k)$ : we will show that $N$ is nef. This implies that for any map $f: \mathbb{P}^{1} \rightarrow M_{i}$, deg $f^{*} N \geq 0$. Hence for any section $s$ of $\pi$ we deduce that $\operatorname{deg} s^{*} \operatorname{det}\left(T M_{i}^{v}\right) \geq-k$.

Let $\lambda_{1}, \ldots, \lambda_{u}$ be the weights of the $G$-module $\Gamma(G / P, \operatorname{det}(T G / P))$, counted with multiplicities (so that $u=\operatorname{dim} \Gamma\left(G / P, \operatorname{det}(T G / P)\right.$ ). Since $\pi_{*} \operatorname{det}\left(T M_{i}^{v}\right)$ is the vector bundle

$$
\Gamma(G / P, \operatorname{det}(T G / P)) \times \mathbb{C}^{*}\left(\mathbb{C}^{2}-0\right),
$$

we get

$$
\pi_{*} N=\bigoplus_{j=1}^{r} O\left(\left\langle\lambda_{j}, \omega_{i}^{\vee}\right\rangle+k\right) .
$$

For the lowest weight $-\lambda_{P}$ of $\Gamma(G / P, \operatorname{det}(T G / P))$, we have $\left\langle-\lambda_{P}, \omega_{i}^{\vee}\right\rangle=-k$. Therefore all the line bundles in (1) have non-negative degree. But then $N$ is basepoint free, hence nef. This concludes the proof.

Since $\operatorname{det}\left(T M_{i}^{v}\right)$ is relatively ample, we get an embedding $M_{i} \rightarrow \mathbb{P}\left(\pi_{*} N\right)^{\vee}$. Let us decompose $\Gamma(G / P, \operatorname{det}(T G / P))$ as $\bigoplus_{j \geq 0} E_{j}$, where our $\mathbb{C}^{*}$ has weight $j-k$ on $E_{j}$. We have an inclusion

$$
\left(G / P \cap \mathbb{P} E_{0}^{\vee}\right) \times \mathbb{P}^{1} \subset M_{i} \subset \mathbb{P}\left(\pi_{*} N\right)^{\vee} .
$$

Proposition 4.4. If $s$ is a section of $\pi$ such that $\operatorname{deg} s^{*} \operatorname{det}\left(T M_{i}^{v}\right)=-k$, there exists $x_{0} \in G / P \cap \mathbb{P} E_{0}^{\vee}$ such that $s(u)=\left(u, x_{0}\right) \in \mathbb{P}^{1} \times\left(G / P \cap \mathbb{P} E_{0}^{\vee}\right)$ for all $u \in \mathbb{P}^{1}$. 
Proof. The space of sections of $N$ identifies with $\bigoplus E_{j} \otimes S^{j} \mathbb{C}^{2}$. Let $f: M_{i} \rightarrow$ $\mathbb{P}\left(\bigoplus E_{j}^{\vee} \otimes S^{j} \mathbb{C}^{2}\right)$ be the morphism defined by $N$. Since $f^{*} O(1)=N$, if the degree of $s^{*} \operatorname{det}\left(T M_{i}^{v}\right)$ is $-k$ then $f \circ s$ is constant.

Note that for $u \neq v \in \mathbb{P}^{1}$, we have $f\left(\pi^{-1}(u)\right) \cap f\left(\pi^{-1}(v)\right) \subset \mathbb{P} E_{0}^{\vee}$. Since $f \circ s$ is constant, this implies that for all $u \in \mathbb{P}^{1}, s(u)$ belongs to $\mathbb{P}^{1} \times \mathbb{P} E_{0}^{\vee}$ and that the induced morphism $\mathbb{P}^{1} \rightarrow \mathbb{P} E_{0}^{\vee}$ is constant. The proposition is proved.

Proposition 4.1 is a consequence of the last two results and the following lemma 4.5 (in fact, we still have to prove that $S$ is reduced; this will be a consequence of the following argument).

Let us compute the constant term of $S\left(\omega_{i}^{\vee}\right)$, the Seidel element corresponding to the loop $\omega_{i}^{\vee}$. With the notations of [9], this is $S_{0}(\pi)$ where $\pi: M_{i} \rightarrow \mathbb{P}^{1}$ is our fibration. According to the remark before [14, Proposition 7.11], we can compute $S_{0}(\pi)$ as the push-forward $\left(e v_{z_{0}}\right)_{*} S$ of the space $S$ of sections of $\pi$ of degree $-k$; by proposition 4.1 we deduce $S_{0}(\pi)=d \cdot \sigma^{P}\left(v_{f(i)}\right)$, where $d \geq 1$, with $d>1$ in case $S$ is not reduced.

We can now easily complete the proof of Theorem 2. We can write any class $x \in Q H^{*}(G / P)$ as $x=\sum x_{w, I} \sigma^{P}(w) q^{I}$ for some integers $x_{w, I}$. We let $|x|:=\sum x_{w, I}$. By formula $[9,8.6 .4]$ and since $M_{i}$ is an algebraic variety, all the coefficients $S\left(\omega_{i}^{\vee}\right)_{w, I}$ are non-negative. Moreover we know that $S\left(\omega_{i}^{\vee}\right)_{v_{f(i)}, 0}=d$. From Theorem 1, and since the product of any two effective classes is again effective, it follows that for any effective class $x$ in $Q H^{*}(G / P)$, we have $\left|x * S\left(\omega_{i}^{\vee}\right)\right| \geq|x|$. Since $S\left(\omega_{i}^{\vee}\right)$ is unipotent, this forces $\left|S\left(\omega_{i}^{\vee}\right)\right|=1$, hence $S\left(\omega_{i}^{\vee}\right)=\sigma^{P}\left(v_{f(i)}\right)$. In particular $d=1$, which also completes the proof of proposition 4.1.

All that remains to prove is the following claim:

Lemma 4.5. Let $L$ be a very ample homogeneous line bundle on $G / P$. Let $G / P \subset$ $\mathbb{P} \Gamma(G / P, L)^{\vee}$ be the corresponding embedding. Let $E_{0} \subset \Gamma(G / P, L)$ be the lowest weight space for a one parameter subgroup of $G$ corresponding to a simple root $\alpha_{i}$, and $P_{i} \subset G$ the corresponding parabolic subgroup. Then $\left(G / P \cap \mathbb{P} E_{0}^{\vee}\right)_{\text {red }}$ is the closed $P_{i}$-orbit in $G / P$; its homology class is $\sigma^{P}\left(v_{f(i)}\right)$.

Proof. Let $x \subset \Gamma(G / P, L)^{\vee}$ be the highest weight line. Then $x \in G / P$. Moreover, $\Gamma(G / P, L)^{\vee}$ is generated as a vector space by $U(\mathfrak{g}) . x$, where $U(\mathfrak{g})$ denotes the enveloping algebra of $\mathfrak{g}$. Consider $\mathfrak{l}=\mathfrak{t} \oplus \bigoplus_{\alpha:\left\langle\alpha, \omega_{i}^{\vee}\right\rangle=0} \mathfrak{g}_{\alpha}$, a Levi subalgebra of $\mathfrak{p}_{i}=\operatorname{Lie}\left(P_{i}\right)$; let $L \subset G$ be the corresponding subgroup. By definition of $E_{0}$, its dual $E_{0}^{\vee}$ is generated by the lines $U(\mathfrak{l}) . x$. This implies that $E_{0}^{\vee}$ is an irreducible $L$-module.

The closed $L$-orbit $L . x$ is contained in $G / P \cap \mathbb{P} E_{0}^{\vee}$. We claim that they are equal. In fact, let $O$ be any $L$-orbit in $G / P \cap \mathbb{P} E_{0}^{\vee}$. We have $L . x \subset \bar{O}$. Moreover,

$$
T_{x} L \cdot x=\bigoplus_{\left\langle\alpha, \omega_{i}^{\vee}\right\rangle=0} \mathfrak{g}_{\alpha} \cdot x=T_{x} \bar{O} .
$$

Thus $L . x=\bar{O}$. 
Finally, in order to identify the Schubert class $[L . x]$, we consider the incidence diagram

$$
\begin{aligned}
& G /\left(P \cap P_{i}\right) \rightarrow G / P \\
& \quad \downarrow p_{i} \\
& G / P_{i}
\end{aligned}
$$

Let $w \in W_{P_{i}}$ be the element corresponding to a fiber of $p_{i}$ (that is, the minimum length representative of the class modulo $W_{P}$ of the longest element in $\left.W_{P_{i}}\right)$, and let $v^{P \cap P_{i}} \in W$ represent the open orbit in $G /\left(P \cap P_{i}\right)$. The element in $W^{P_{i}}$ corresponding to the open orbit in $G / P_{i}$ is $v_{i}$, and therefore we have the relation $v^{P \cap P_{i}}=v_{i} w$, which implies, since $v_{i}^{-1}=v_{f(i)}$, the relation $w=v_{f(i)} v^{P \cap P_{i}}$. Let $F_{i}$ denote a fiber of $p_{i}$ in $G /\left(P \cap P_{i}\right)$. We deduce that $[F]$ identifies by Poincare duality with $\sigma^{P}\left(w_{0} v_{f(i)} w_{0}\right)=\sigma^{P}\left(v_{i}\right)$. Since the projection $G /\left(P \cap P_{i}\right) \rightarrow G / P$ restricts to an isomorphism on $F_{i}$, we deduce that $[L . x]$ identifies with $\sigma^{P}\left(v_{i}\right)$.

\section{References}

[1] N. Bourbaki, Groupes et algèbres de Lie, Hermann (1954).

[2] P.-E. Chaput, L. Manivel, and N. Perrin, Quantum cohomology of minuscule homogeneous spaces II : Hidden symmetries, Int. Math. Res. Notices 22 (2007)

[3] - Quantum cohomology of minuscule homogeneous spaces III : semi-simplicity and consequences (2007). ArXiv:math.AG/0710.1224.

[4] B. Kostant and S. Kumar, The nil Hecke ring and cohomology of $G / P$ for a Kac-Moody group $G$, Proc. Nat. Acad. Sci. U.S.A. 83 (1986), no. 6, 1543,1545.

[5] S. Kumar, Kac-Moody groups, their flag varieties and representation theory, number 204 in Progress in Mathematics, Birkhäuser Boston, Inc., Boston, MA (2002).

[6] T. Lam, Schubert polynomials for the affine Grassmannian, J. Amer. Math. Soc. 21 (2008) 259,281 .

[7] T. Lam and M. Shimozono, Quantum cohomology of G/P and homology of affine Grassmannian (2007). ArXiv:0705.1386.

[8] P. Magyar, Notes on Schubert classes of a loop group (2007). ArXiv:0705.3826.

[9] D. McDuff and D. Salamon, $J$-holomorphic curves and symplectic topology, 52, American Mathematical Society, Providence, RI (2004).

[10] L. Mihalcea, On equivariant quantum cohomology of homogeneous spaces: Chevalley formulae and algorithms, Duke Math. J. 140 (2007) 321,350.

[11] D. Peterson, Quantum cohomology of $G / P$ (1997).

[12] A. Postnikov, Symmetries of Gromov-Witten invariants, Contemp. Math. 276 (2001) 251,258.

[13] - Affine approach to quantum Schubert calculus, Duke Math. J. 128 (2005), no. 3, 473,509.

[14] P. Seidel, $\pi_{1}$ of symplectic automorphism groups and invertibles in quantum homology rings, Geom. Funct. Anal. 7 (1997), no. 6, 1046,1095.

Laboratoire de Mathématiques Jean Leray, UMR 6629 du CNRS, UFR Sciences et TechNiques, 2 Rue de la Houssinière, BP 92208, 44322 Nantes Cedex 03, France.

E-mail address: pierre-emmanuel.chaput@math.univ-nantes.fr

Institut Fourier, UMR 5582 du CNRS, Université de Grenoble I, BP 74, 38402 SaintMartin d'Hères, France.

E-mail address: Laurent.Manivel@ujf-grenoble.fr

Institut de Mathématiques, Université Pierre et Marie Curie,, Case 247, 4 Place Jussieu, 75252 PARIS Cedex 05, France, and, Hausdorff Center for Mathematics,, Universität Bonn, Landwirtschaftskammer (Neubau) Endenicher Allee 60, 53115 Bonn, Germany.

E-mail address: nperrin@math.jussieu.fr 